University of Nebraska - Lincoln

DigitalCommons@University of Nebraska - Lincoln

\title{
5-27-1985
}

\section{Relativistic Plasma-Wave Excitation by Collinear Optical Mixing}

\author{
C.E. Clayton \\ University of California, Los Angeles \\ C. Joshi \\ University of California, Los Angeles \\ C. Darrow \\ University of California, Los Angeles \\ Donald P. Umstadter \\ University of Nebraska-Lincoln, donald.umstadter@unl.edu
}

Follow this and additional works at: https://digitalcommons.unl.edu/physicsumstadter

Part of the Physics Commons

Clayton, C.E.; Joshi, C.; Darrow, C.; and Umstadter, Donald P., "Relativistic Plasma-Wave Excitation by Collinear Optical Mixing" (1985). Donald Umstadter Publications. 38.

https://digitalcommons.unl.edu/physicsumstadter/38

This Article is brought to you for free and open access by the Research Papers in Physics and Astronomy at DigitalCommons@University of Nebraska - Lincoln. It has been accepted for inclusion in Donald Umstadter Publications by an authorized administrator of DigitalCommons@University of Nebraska - Lincoln. 


\title{
Relativistic Plasma-Wave Excitation by Collinear Optical Mixing
}

\author{
C. E. Clayton, C. Joshi, C. Darrow, and D. Umstadter \\ Electrical Engineering Department, University of California, Los Angeles, California 90024 \\ (Received 8 March 1985)
}

\begin{abstract}
The relativistic plasma wave excited when the frequency difference between two copropagating $\mathrm{CO}_{2}$ laser beams equals the plasma frequency is detected for the first time. The plasma-wave frequency, wave number, spatial extent, and saturation time are directly measured by use of 7-mrad, collective, ruby Thomson scattering and the forward-scattered ir spectrum. The wave amplitude $\tilde{n} / n_{0}$ is inferred to be (1-3)\% which gives a longitudinal electric field of 0.3 to $1 \mathrm{GV} / \mathrm{m}$ at a laser intensity of $1.7 \times 10^{13} \mathrm{~W} / \mathrm{cm}^{2}$, in reasonable agreement with theory.
\end{abstract}

PACS numbers: $52.35 . \mathrm{Fp}, 52.35 . \mathrm{Mw}, 52.40 . \mathrm{Nk}$

The excitation of plasma waves through the method of optical mixing has been of interest to plasma physicists for many years. In this process, two electromagnetic waves interfere to resonantly excite a wave in a plasma. $^{1-4}$ Applications of optical mixing have been envisioned or found in such areas as plasma diagnostics, ${ }^{1,5,6}$ plasma heating, ${ }^{2,3}$ and the study of plasmawave physics. ${ }^{7}$ The work reported here is part of an ongoing effort in theory, ${ }^{8,9}$ simulation, ${ }^{10}$ and experiment ${ }^{11}$ to study the physics relevant to a new application, namely, a particle accelerator based on the plasma beat-wave accelerator concept. ${ }^{8}$ The motivation lies in the fact that electron plasma waves can support electric fields up to orders of magnitude larger than the fields in conventional accelerators (cf. $20 \mathrm{MV} / \mathrm{m}$ for the Stanford linac). Moreover, these fields can be made to propagate at a phase velocity $v_{\phi} \simeq c$ so that acceleration can occur to relativistic energies. This last, most crucial property of the field requires the two electromagnetic waves to propagate parallel to each other. This Letter presents the first direct observation of the relativistic electron plasma wave excited by this collinear optical mixing process. It is confirmed experimentally that $v_{\phi} \simeq c$ and that the electric field of the wave is indeed large $(0.3-1.0 \mathrm{GV} / \mathrm{m})$.

For this experiment, a $\mathrm{CO}_{2}$ laser is used which delivers $16 \mathrm{~J}$ in a pulse of 2 nsec full width at half maximum with a $1-n s e c$ rise time. The laser can be operated either on a single wavelength $(10.59 \mu \mathrm{m})$ or on the two wavelengths 9.56 and $10.59 \mu \mathrm{m}$ with line energies of 4 and $12 \mathrm{~J}$, respectively. The beam is focused with an $f / 7.5$ optics (vacuum intensity $\simeq 1.7 \times 10^{13} \mathrm{~W} / \mathrm{cm}^{2}$ ) into a chamber containing about 1 Torr of hydrogen gas which has been preionized by an arc discharge. The laser-heated plasma has a variable density (as discussed later) near $10^{17} \mathrm{~cm}^{-3}$ and an electron temperature of $30 \pm 10 \mathrm{eV}$.

If $\left(\omega_{0}, \mathbf{k}_{0}\right)$ and $\left(\omega_{1}, \mathbf{k}_{1}\right)$ are the frequencies and wave vectors of the 9.56- and 10.59- $\mu \mathrm{m}$ lines, respectively, then an electron plasma wave $\left(\omega_{p}, \mathbf{k}_{p}\right)$ can be resonantly excited ${ }^{2}$ if $\omega_{0}-\omega_{1} \equiv \Delta \omega=\omega_{p}$ and $\mathbf{k}_{0}-\mathbf{k}_{1}$ $\equiv \Delta \mathbf{k}=\mathbf{k}_{p}$. For $\omega_{0}, \omega_{1} \gg \Delta \omega, \omega_{p e}$ [where $\omega_{p e}=(4 \pi$ $\left.\times n_{0} e^{2} / m\right)^{1 / 2}$ is the plasma frequency and $n_{0}$ is the average plasma density], we have that $k_{0}, k_{1}>\Delta k$ (for collinear optical mixing) and that $\Delta \omega / \Delta k=\omega_{p} / k_{p}$ $\equiv v_{\phi} \simeq c\left(1-\Delta \omega^{2} / \omega_{0}^{2}\right)^{1 / 2}$. In our experiment, $(\Delta \omega /$ $\left.\omega_{0}\right)^{-1} \simeq 10.3$ and $v_{\phi} / c=0.995$. Also, since $k_{p}$ is small, the thermal correction to the plasma-wave frequency can be neglected so that $\omega_{p} \simeq \omega_{p e}$. According to the theory of Rosenbluth and Liu, ${ }^{2}$ the plasma wave will grow until it saturates because of relativistic detuning, at which point the amplitude will fall again. This saturation level $\left(\tilde{n} / n_{0}\right)_{\max }$ and the time to reach saturation $t_{s}$ were recently rederived to take into account the laser rise time. ${ }^{10}$ With use of the formulas of Ref. 10, the peak line intensities of about $I_{0}=4.4 \times 10^{12} \mathrm{~W} / \mathrm{cm}^{2}(9.56 \mu \mathrm{m})$ and $I_{1}=1.7 \times 10^{13}$ $\mathrm{W} / \mathrm{cm}^{2}(10.59 \mu \mathrm{m})$ should yield $\left(\tilde{n} / n_{0}\right)_{\max } \simeq 8 \%$ and $t_{s}=500 \mathrm{psec}$, under the assumption of a linear rise of the laser field amplitude. If a linear rise of the field intensity is assumed, the maximum wave amplitude and the time to saturation will be about $9 \%$ and 300 psec, respectively. The above theoretically estimated plasma-wave amplitude should be easily detected by collective Thomson scattering.

For the two laser frequencies used, the plasma density must be adjusted to $1.15 \times 10^{17} \mathrm{~cm}^{-3}$ for the resonant excitation of plasma waves to occur. Experimentally, this adjustment is accomplished by variation of the fill pressure, arc voltage, or relative timing of the arc discharge with respect to the laser pulse. With the $\mathrm{CO}_{2}$ laser operating in the single-frequency mode, the frequencies of the plasma waves with $\mathbf{k} \simeq 2 \mathbf{k}_{1}$ excited by the stimulated-Raman-backscatter instability ${ }^{12}$ are detected with use of $7.5^{\circ}$ collective ruby-laser Thomson scattering. This gives the local plasma density to within a few percent uncertainty through the plasma-wave dispersion relation. Some spectra taken under various conditions are shown in Fig. 1. In this manner the experimental parameters are established which reproducibly give us the resonant density. As discussed later, the measurement of forward-scattered, frequency-shifted $\mathrm{CO}_{2}$ light provides an independent, on-line confirmation of the presence of the resonant 

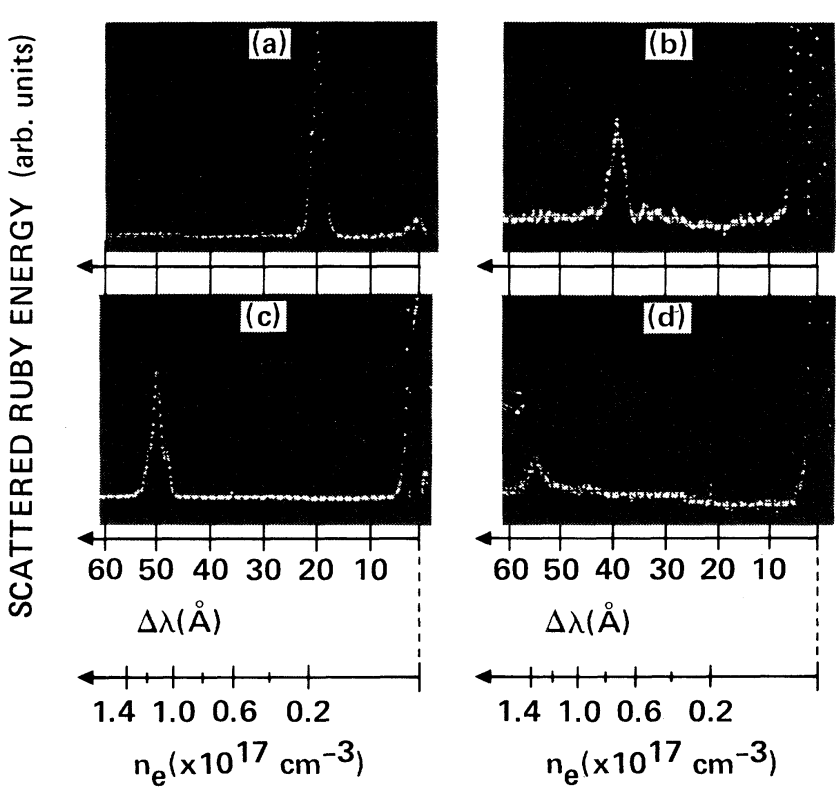

FIG. 1. Frequency spectra of waves with $\mathbf{k}=2 \mathbf{k}_{1}$. The peaks on the right in each spectrum are due to ion waves from Brillouin scattering and so are essentially unshifted in frequency $(\Delta \lambda=-0.1 \AA)$. The peaks on the left are from Raman plasma waves and are thus indicative of the local plasma density (lower scale), which was varied in (a) - (d) by variation of the arc discharge parameters.

density under two-frequency illumination. With the resonant plasma density tuned in, the beat-excited "fast wave" can now be driven by running the $\mathrm{CO}_{2}$ laser in the two-frequency mode.

The collective-Thomson-scattering setup for diagnosing the fast wave is different from that used to detect the Raman-generated waves. Because the fast wave has a very small $k\left[k_{p} \simeq\left(\Delta \omega / \omega_{0}\right) k_{0}<<2 k_{1}\right]$, the scattering angle is correspondingly small-only 7 mrad. A novel optical system was devised ${ }^{13}$ which brings the scattered and unscattered ruby beams to different foci, separated by $1 \mathrm{~mm}$ per mrad of scattering angle. An optical fiber $(1 \mathrm{~mm}$ diameter $)$ placed $7 \mathrm{~mm}$ from the unscattered beam thus collects the 7-mrad scattered light. By translating the fiber parallel to $\mathbf{k}_{p}$, one maps out the angular distribution of scattered light and hence the $k$ spectrum of the plasma waves. As shown in Fig. 2(a), there is a definite peak in the $k$ spectrum centered at the expected value of $k_{p}=\Delta k$. Figure 2(b) shows the frequency spectrum of the scattered light indicating that the plasma frequency is centered at $\omega_{p}=\Delta \omega$, as it should for a wave with $v_{\phi} \simeq c$. It is also noted that Thomson-scattered ruby light is only detected when the laser operates on two frequencies and when the plasma is tuned to the resonant density. Clearly, the wave evident in Fig. 2 is the resonantly excited electron plasma wave produced by

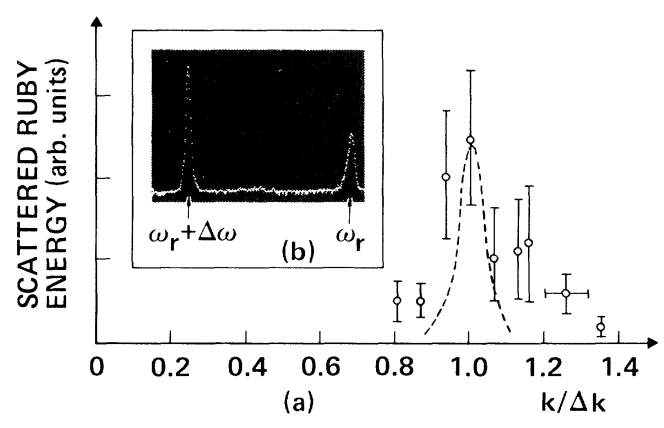

FIG. 2. (a) Electron-plasma-wave $k$ spectrum. Each point is the average, with standard deviation, of three or more shots (as are the points in Figs. 3 and 4). The horizontal bar indicates the window of measurement and the dashed curve represents the instrument function (see Ref. 13). (b) Frequency spectrum with stray ruby light $\left(\omega_{r}\right)$ and its satellite shifted by the plasma-wave frequency.

optical mixing of the two laser lines. The lack of detectable scattered light with single-frequency operation indicates that the noise fluctuations near $(\omega, k)=(\Delta \omega, \Delta k)$ are less than $3 \times 10^{-3} n_{0}$ in amplitude, the detection threshold. It also indicates that the product of intensity times density scale length is below the forward-Raman-scattering threshold. With the assumption of a parabolic density profile, $n(x)$ $=n_{0}\left(1-x^{2} / L^{2}\right)$, the threshold scale length at the peak laser intensity ${ }^{14}$ is $L \simeq 7.2 \mathrm{~mm}$, which can be considered as an upper bound of the actual density scale length. A lower bound can be estimated by applying the same consideration to the observation of Raman backscatter (Fig. 1). Again with the assumption of a parabolic density profile, being above threshold $^{14}$ implies that $L>0.2 \mathrm{~mm}$. As discussed later, a scale length even as long as the upper estimate may be a significant limitation on the length of the resonant region.

Another consequence of collinear optical mixing is the production of $\omega_{p}$-downshifted (Stokes) and $\omega_{p^{-}}$ upshifted (anti-Stokes) sidebands of the laser frequencies. Indeed, when a small portion of the transmitted light is analyzed, two Stokes and one anti-Stokes sidebands are observed. Since the sidebands are seen only under two-wavelength illumination and when $\omega_{p e}$ $\simeq \Delta \omega$, they provide shot-by-shot confirmation of the resonance condition. The relative amplitudes of the forward-going electromagnetic sidebands are plotted in Fig. 3(a). Figure 3(b) shows the spectral detail of the anti-Stokes line obtained with a monochromator. At the laser intensities used, the sidebands are produced by Thomson scattering of the pump waves from the driven plasma wave, exactly analogous to the rubyscattering process except that there is a small, unavoidable $k$ mismatch in the $\mathrm{CO}_{2}$-scattering case. ${ }^{3}$ This correspondence of the $\mathrm{CO}_{2}$ and ruby scattering is 


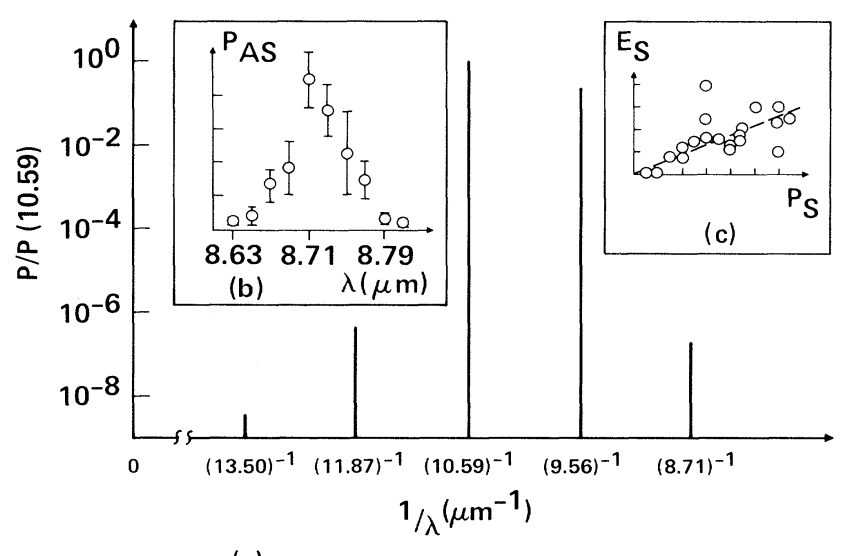

(a)

FIG. 3. (a) A bar graph of the average relative powers in the two laser lines and in the three sidebands. The second Stokes sideband at $13.5 \mu \mathrm{m}$ was only occasionally seen above the detection threshold, and so its bar is an upper bound. (b) Spectral detail of the anti-Stokes line, the width of which is consistent with the spectral content of the pump lines. (c) Scattered ruby energy $\left(E_{s}\right)$ vs scattered $\mathrm{CO}_{2}$ power in the Stokes line $\left(P_{s}\right)$. Each point is one laser shot.

borne out experimentally, as shown in Fig. 3(c). A straight line here would indicate that the ruby and $\mathrm{CO}_{2}$ scattered light are both proportional to the intensity of the plasma wave. Although this is true at low values of $P_{s}$, there is a significant deviation from proportionality at higher values of $P_{s}$ which is not yet understood. The second Stokes line is most likely due to Thomson scattering of the $10-\mu \mathrm{m}$ pump from the second harmonic of the fast wave (rescattering of the first Stokes line should be undetectable). If so, the amplitude of the second Stokes line can indicate how close the wave is to wave breaking, where the second harmonic becomes comparable to the fundamental. An attempt to measure the $\left(2 \omega_{p}, 2 k_{p}\right)$ harmonic with ruby scattering failed, implying that its power is down at least 100 times from the fundamental, which is consistent with the second Stokes data, which is also down more than 100 times from its fundamental.

The amplitude, length, and time duration are still needed to complete the picture of the fast wave. The length of the plasma wave was measured in the following way. The ruby beam is cylindrically focused and samples a length $L_{r} \simeq 1 \mathrm{~cm}$, much longer than the length $L_{p}$ of the plasma wave. By masking the beam with a $460-\mu \mathrm{m}$ slit that corresponds to about five plasma-wave wavelengths and scanning the slit across the width of the beam, one maps out the region occupied by the plasma wave, giving $L_{p}=1.8 \mathrm{~mm}$, as shown in Fig. 4(a). Since the measured focal depth of the laser beam is quite long (about $8 \mathrm{~mm}$ ), the limitation on the length of the plasma wave is suspected to

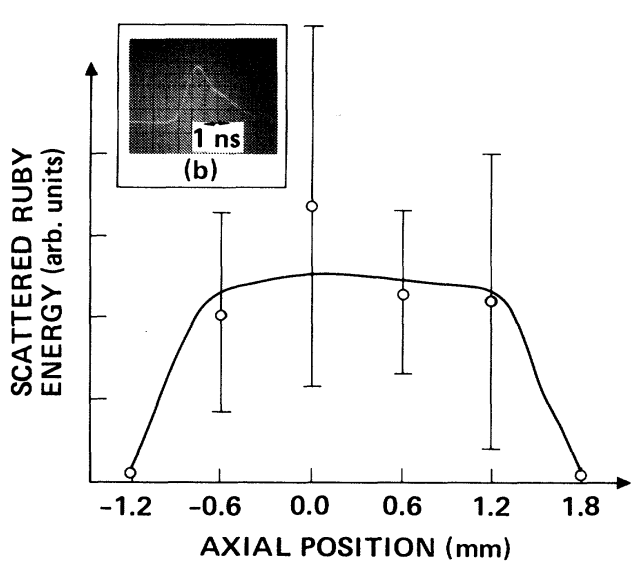

(a)

FIG. 4. (a) Scattered ruby energy or plasma-wave amplitude (squared) vs axial position, where zero is the approximate location of the $\mathrm{CO}_{2}$ best focus. (b) Pulse shape of the $11.87-\mu \mathrm{m}$ Stokes radiation.

be due to plasma inhomogeneity. As discussed before, Raman-threshold considerations indicate that the scale length satisfies $0.2<L<7.2 \mathrm{~mm}$. A parabolic density scale length of $7 \mathrm{~mm}$ is consistent with the observed $1.8-\mathrm{mm}$ resonant length if we assume that the density must be matched to within about $2 \%$.

The rise time and pulse width of the Stokes line should be characteristic of the corresponding properties of the plasma wave. Figure 4(b) shows a typical fast-rising Stokes signal with a rise time $(5 \%$ to $95 \%$ of peak) of 700-800 psec. After deconvolution of the detector-scope response time, ${ }^{15}$ the actual rise time becomes $360-530$ psec, a range which is consistent with the theoretically predicted times to saturation given earlier. The lifetime $\tau_{p}$ of the Stokes line and thus of the plasma wave is $\tau_{p} \simeq 1 \mathrm{nsec}$ full width at half maximum.

The wave amplitude is determined by making an absolute measurement of the ratio of the scattered $E_{p}$ to the unscattered $E_{r}$ ruby energy and using the Bragg scattering formula, ${ }^{16}$

$$
\frac{\left(E_{p} / \tau_{p}\right)}{\left(E_{r} / \tau_{r}\right)}=\frac{P_{s}}{P_{0}}=\left(\frac{\pi}{2} \frac{\tilde{n}}{n_{0}} \frac{n_{0}}{n_{\mathrm{cr}}} \frac{d}{\lambda_{r}}\right)^{2} \frac{L_{p}}{L_{r}},
$$

where $P_{0}$ and $P_{s}$ are the peak incident and scattered powers, respectively, $n_{\mathrm{cr}}$ is the critical density for ruby light (wavelength $\lambda_{r}$ ), $\tau_{r}$ is the pulse width of the ruby light (about 20 nsec full width at half maximum), and the other factors are as defined before. The effective width $^{17}$ of the plasma wave, $d$, is estimated as $120 \mu \mathrm{m}$ or about one-half of the $\mathrm{CO}_{2}$ spot size. It is found experimentally that $E_{p} / E_{r}=10^{-10}-10^{-9}$, which with the other parameters in Eq. (1) yields $\left(\tilde{n} / n_{0}\right)_{\max }$ $=(1-3) \%$. From Poisson's equation, this corresponds 
to a longitudinal electric field of $0.3-1.0 \mathrm{GV} / \mathrm{m}$. This estimate is somewhat smaller than the fluid-theory prediction. However, some higher-order processes have been neglected in the theory. The fast wave can be, in effect, damped by such wave-wave interactions as quasiresonant mode coupling ${ }^{18}$ through the presence of a large-amplitude ion wave (from Brillouin scattering). Electron-ion collisions may also be important as the collisional damping rate is on the order of the effective fast-wave growth rate. The effects of finite damping were tested by adding a phenomenological damping term to the Rosenbluth and Liu wave equation, and it was found numerically that $\left(\tilde{n} / n_{0}\right)_{\max }$ decreased by about $15 \%$ and $t_{s}$ increased by about $40 \%$ for an amplitude damping rate of $5 \times 10^{-4} \omega_{p e}$. Other effects neglected in theory are finite laser bandwidth, nonideal laser spatial-temporal coherence, and density mismatches due to Brillouin ion waves.

In conclusion, the high-phase-velocity electron plasma wave excited by collinear optical mixing has been detected directly for the first time. The frequency, wave number, spatial extent, saturation time, and peak amplitude were all measured experimentally and were found to be in reasonable agreement with the expectations from theory. Finally, with a modest laser intensity of $1.7 \times 10^{13} \mathrm{~W} / \mathrm{cm}^{2}$, the driven plasma wave has a maximum measured longitudinal electric field of 1 $\mathrm{GV} / \mathrm{m}$, offering interesting possibilities for collective particle acceleration.

We acknowledge useful conversations with W. Mori, T. Katsouleas, J. M. Dawson, and F. F. Chen. This work was supported by the U. S. Department of Energy under Contract No. DE-AT03-83ER40120, the Na- tional Science Foundation under Grant No. ECS 8310972, and the Lawrence Livermore National Laboratory University Research Program.

${ }^{1}$ N. M. Kroll et al., Phys. Rev. Lett. 13, 83 (1964).

${ }^{2}$ M. N. Rosenbluth and S. C. Liu, Phys. Rev. Lett. 29, 701 (1972).

${ }^{3}$ B. I. Cohen et al., Phys. Rev. Lett. 29, 581 (1972).

${ }^{4}$ C. M. Tang et al., Appl. Phys. Lett. 45, 375 (1984).

${ }^{5}$ B. Amini and F. F. Chen, Phys. Rev. Lett. 53, 1441 (1984).

6B. L. Stansfield et al., Phys. Rev. Lett. 26, 1219 (1971).

${ }^{7}$ C. J. Pawley et al., Phys. Rev. Lett. 49, 877 (1982).

${ }^{8}$ T. Tajima and J. M. Dawson, Phys. Rev. Lett. 43, 267 (1979).

${ }^{9}$ C. Joshi et al., Nature 311, 525 (1984).

${ }^{10}$ D. W. Forslund et al., Phys. Rev. Lett. 54, 558 (1985).

${ }^{11}$ C. Joshi et al., Phys. Rev. Lett. 48, 874 (1982).

${ }^{12}$ D. W. Forslund et al., Phys. Fluids 18, 1002 (1975).

${ }^{13}$ C. E. Clayton et al., to be published.

${ }^{14} \mathrm{H}$. Figueroa, private communication.

${ }^{15}$ F. E. Terman and J. M. Pettit, Electronic Measurements (McGraw-Hill, New York, 1952), pp. 259-260.

${ }_{16}$ R. E. Slusher and C. M. Surko, Phys. Fluids 23, 472 (1980).

${ }^{17}$ Equation (1) assumes that $\tilde{n} / n_{0}$ is constant over a distance $d$. However, in optical mixing $\tilde{n} / n_{0}$ has the same radial profile as the pump intensity (nearly Gaussian) up until the time of saturation. Since we are interested in the peak $\tilde{n} / n_{0}$, we take $d$ as some effective width which should be about half the $\mathrm{CO}_{2}$ spot size or about $120 \mu \mathrm{m}$.

18P. K. Kaw et al., Phys. Fluids 16, 1967 (1973). 


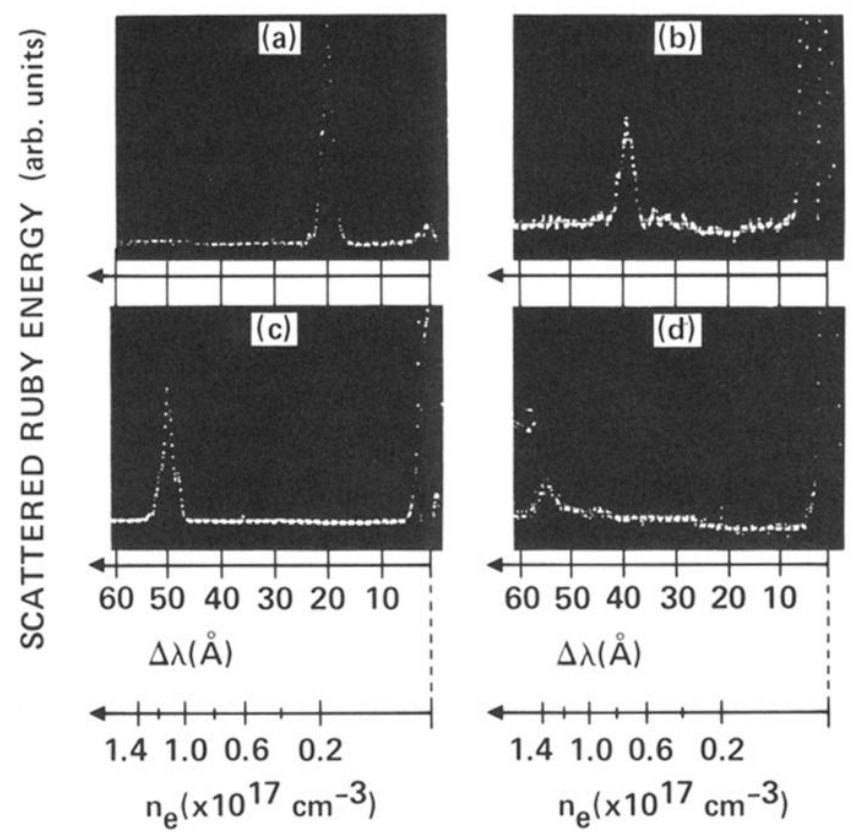

FIG. 1. Frequency spectra of waves with $k \simeq 2 k_{1}$. The peaks on the right in each spectrum are due to ion waves from Brillouin scattering and so are essentially unshifted in frequency $(\Delta \lambda \simeq-0.1 \AA)$. The peaks on the left are from Raman plasma waves and are thus indicative of the local plasma density (lower scale), which was varied in (a) - (d) by variation of the arc discharge parameters. 


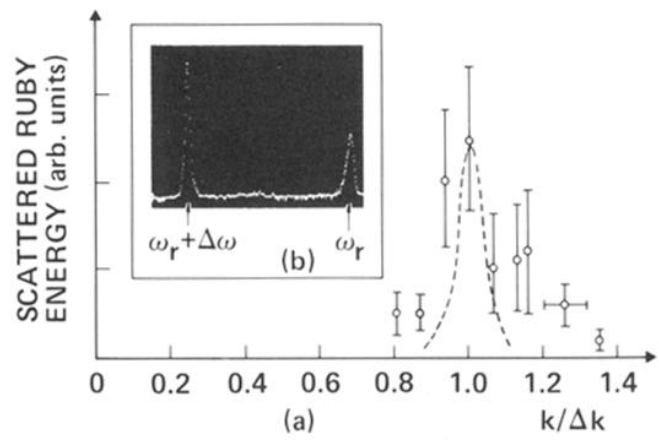

FIG. 2. (a) Electron-plasma-wave $k$ spectrum. Each point is the average, with standard deviation, of three or more shots (as are the points in Figs. 3 and 4). The horizontal bar indicates the window of measurement and the dashed curve represents the instrument function (see Ref. 13). (b) Frequency spectrum with stray ruby light $\left(\omega_{r}\right)$ and its satellite shifted by the plasma-wave frequency. 


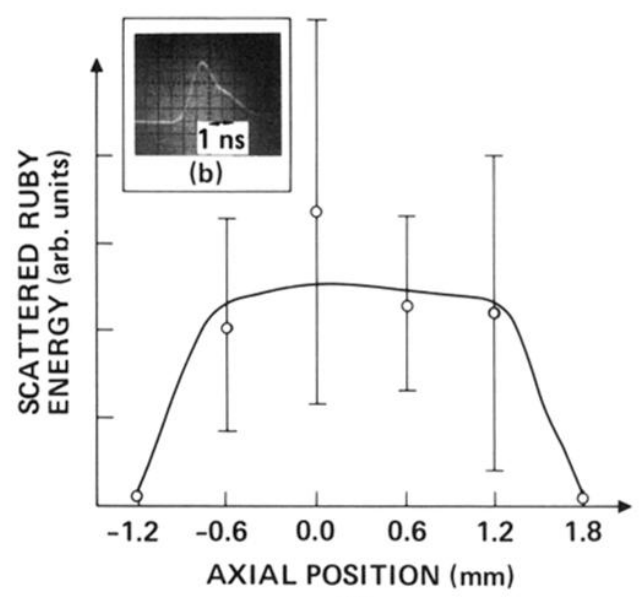

(a)

FIG. 4. (a) Scattered ruby energy or plasma-wave amplitude (squared) vs axial position, where zero is the approximate location of the $\mathrm{CO}_{2}$ best focus. (b) Pulse shape of the $11.87-\mu \mathrm{m}$ Stokes radiation. 